\title{
Espiritualidade em cuidados paliativos no Brasil: revisão integrativa de literatura
}

\section{Spirituality in palliative care in Brazil: an integrative literature review}

\author{
Mary Esperandio* \\ Carlo Leget**
}

Resumo: Considerada uma dimensão essencial dos Cuidados Paliativos (CP), a espiritualidade tem sido objeto de rigorosa investigação científica nos Estados Unidos e Europa. No Brasil, a implementação de serviços de $\mathrm{CP}$ e a discussão sobre a integração da espiritualidade neste contexto são recentes. Este estudo apresenta o atual estado da arte sobre o tema. Foram realizadas buscas no Portal de Periódicos CAPES, Biblioteca Virtual em Saúde, SciELO e PUBMED. Ao todo, 25 estudos foram selecionados para análise, sendo 17 com foco central sobre espiritualidade nos CP. A literatura evidencia percepção crescente acerca do papel da espiritualidade nesse contexto; necessidade de pesquisas teoricamente fundadas e de modelos práticos de cuidado espiritual; carência na formação profissional em relaçáo ao tema e desconhecimento sobre a importância da assistência espiritual especializada. Para o avanço do conhecimento nesse campo, futuros estudos devem considerar tais lacunas e potencialidades.

Palavras-chave: Cuidados paliativos. Espiritualidade. Saúde. Cuidado espiritual.

Abstract: Considered to be one of the essential dimensions of Palliative Care (PC), spirituality has undergone rigorous scientific research in the United States and Europe. In Brazil, the implementation of PC services and the discussion on the integration of spirituality in this context are relatively recent. This study presents the current state of the art on this subject. Searches were carried out in the CAPES Journals Portal, Virtual Health Library, SciELO and PUBMED. Twenty-five studies were selected for analysis, and 17 had a central focus on spirituality in PC. The literature evidences a growing understanding about the role of spirituality in this context; the need for theoretically founded research and of practical models of spiritual care; lack of professional training in relation to the subject and lack of knowledge about the importance of specialized spiritual assistance. For the advancement of knowledge in this field, future studies should consider these gaps and potentialities.

Keywords: Palliative care. Spirituality. Health. Spiritual care.

* Professora Adjunta no PPG em Teologia e no PPG em Bioética na PUC-PR. Doutora em Teologia (EST). ORCID: 0000-0001-8521-8794 - contato: mary.esperandio@pucpr.br

** Professor titular de "Care Ethics", da University of Humanistic Studies, Utrecht (Países Baixos). Doutor em Teologia (University of Utrecht). ORCID: 0000-0002-6647-8141 - contato: c.leget@uvh.nl 


\section{Introdução}

Em data bastante recente, precisamente em 23/11/2018, o governo brasileiro publicou no Diário Oficial da União a Resolução No 41, de 31/10/2018 (Brasil, 2018) que dispóe sobre as Diretrizes para a organização dos Cuidados Paliativos (CP) no âmbito da saúde pública. Esse fato surge como um divisor de águas nas políticas de cuidado em saúde no Brasil.

Composta por nove artigos, a Resolução estabelece, já em seu Artigo 1º, Parágrafo Único, que "os cuidados paliativos deverão fazer parte dos cuidados continuados integrados ofertados no âmbito da RAS [(Rede de Atenção à Saúde]" (Brasil, 2018, p. 276). O documento segue a definição proposta em 2002 pela World Health Organization WHO (Organização Mundial de Saúde - OMS), explicitando que "cuidados paliativos consistem na assistência promovida por uma equipe multidisciplinar, que objetiva a melhoria da qualidade de vida do paciente e seus familiares, diante de uma doença que ameace a vida, por meio da prevenção e alívio do sofrimento, da identificação precoce, avaliação impecável e tratamento de dor e demais sintomas físicos, sociais, psicológicos e espirituais" (Brasil, 2018, p. 276).

A Resolução lista oito objetivos e 13 princípios que devem nortear a organização dos $\mathrm{CP}$ (Artigo $3^{\circ}$ e $4^{\circ}$ ). Dois desses princípios referem-se às questôes espirituais, destacando a necessidade de "integraçáo dos aspectos psicológicos e espirituais no cuidado ao paciente", bem como a promoção do "alívio do sofrimento espiritual e existencial de pacientes e familiares” (Brasil, 2018, p. 276).

Se por um lado, a publicaçáo desse documento surge como uma promessa de "revolução" nas políticas de saúde e na oferta de serviços de CP no Brasil, por outro, não garante mudança substancial no cenário dos $\mathrm{CP}$ no país, nem a curto, nem a médio prazo, uma vez que, para mudanças reais, seria necessário um contingente significativo de pessoal capacitado, o que ainda levará tempo. $\mathrm{O}$ último levantamento da Academia Nacional de Cuidados Paliativos (ANCP), realizado em agosto de 2018, informa que até aquela data o total de serviços cadastrados era de 177 (ANCP, 2018). Obviamente, é um número pequeno para um país com aproximadamente 210 milhôes de habitantes (IBGE, 2018, online), com um contingente volumoso de pessoas vivendo em condiçóes sociais menos saudáveis, mais vulneráveis, portanto, à doenças graves, e com aumento significativo da população idosa (acima de 60 anos), tendo alcançado em 2017 o patamar de 30,2 milhóes (14,54\%) da população total (IBGE, 2018, online). Em 2012, a porcentagem de idosos era de 7,4\% (IBGE, 2018, online).

$\mathrm{Na}$ categorização da WHO sobre o desenvolvimento de CP no mundo, o Brasil recebeu a classificação no nível 3a (WPCA; WHO, 2014). Nesse grupo, estão os países onde a provisão de $\mathrm{CP}$ é oferecida

[...] de maneira isolada, as ações paliativas são de alcance irregular e não bem estabelecidas, fonte de financiamento dependente de doadores, disponibilidade limitada de morfina e um pequeno número de hospices (locais/instituiçôes voltadas ao cuidado de fim de vida) - que são muitas vezes de natureza domiciliar e relativamente limitados para o tamanho da população (WPCA; WHO, 2014, p. 37). 
Encontram-se nesse nível 74 países. Entre eles Colômbia, Paraguai, Bolívia, Guatemala, Angola, Moçambique e Congo (WPCA; WHO, 2014, p. 37).

Diante da realidade atual e do movimento que se inicia em termos de ampliaçáo na organização de serviços de CP, levanta-se a pergunta acerca de um tema que está na base da filosofia dos CP, isto é, a integração da dimensão espiritual como expressão do cuidado integral. Nesse sentido, não há como conceber um serviço de CP desvinculado do cuidado espiritual, seu "componente fundamental" (Puchalski et al., 2014, p. 642) ou, como afirma Leget $(2018$, p. 2), "a provisão do cuidado espiritual é parte intrínseca às boas práticas de CP”.

Face a isso, emergem perguntas tais como: é a espiritualidade uma dimensão efetivamente integrada nos CP? De que modo ela é (ou pode ser) integrada na prática de cuidado? É possível identificar necessidades espirituais a serem atendidas? Quem deveria fazê-lo? Há profissionais especializados nessa área? Seria o cuidado espiritual uma "especialidade" dentro dos serviços de CP ou algo a ser realizado por toda a equipe? Este estudo teve como objetivo apresentar um mapeamento da E/R nos CP no Brasil a partir da literatura produzida no país, indicando o estado atual, possíveis tendências e alguns dos principais desafios e/ou oportunidades relacionadas à sua efetiva integraçáo nas práticas de cuidado. Em tempos de implementação dos serviços de CP na atenção primária em saúde, a reflexão sobre esse tema é bastante oportuna.

\section{Método}

Trata-se de um estudo de Revisão Integrativa da Literatura. Esse método visa sintetizar o conhecimento construído em torno de uma questáo específica sobre um determinado tema, utilizando como base os resultados de estudos já realizados. Tem como propósito final tanto "a incorporação da aplicabilidade de resultados de estudos significativos" (Souza; Silva; Carvalho, 2010, p. 102) quanto a identificação das lacunas existentes e a definição de agenda de pesquisas futuras (Kastner et al., 2012). Assim, a questão norteadora deste estudo foi a seguinte: "O que os estudos científicos têm mostrado sobre espiritualidade/ religiosidade no contexto dos CP no Brasil? Foram levantados trabalhos publicados em português e inglês, produzidos no Brasil, sem um recorte no período de tempo. Os termos de busca, utilizados em português e inglês, bem como o total de trabalhos capturados em cada base de dados, estão descritos na Tabela 1.

Além dos termos-chave extraídos da pergunta norteadora, duas outras buscas foram realizadas, incluindo os termos "psicologia" e "capelania", ou, "capelães". Tal escolha deu-se em função de assegurar a captura do máximo possível de trabalhos sobre E/R e $\mathrm{CP}$ e, também, para analisar o modo como a Psicologia e a Capelania Hospitalar lidam com a dimensão da $\mathrm{E} / \mathrm{R}$ no contexto específico dos CP no Brasil.

A busca nas bases de dados foi realizada em novembro e dezembro de 2018, no Portal de Periódicos da Coordenação de Aperfeiçoamento de Pessoal de Nível Superior (CAPES); Biblioteca Virtual em Saúde (BVS); Scientific Eletronic Library Online (SciELO); Biblioteca Digital de Teses e Dissertações (BDTD) e PUBMED. A seleção dos estudos deu-se através de um cuidadoso processo de análise descrito na Figura 1. 
Tabela 1 - Termos de busca utilizados nas bases de dados.

Termos de busca

CAPES BVS SciELO PUBMED BDTD Total

\begin{tabular}{|c|c|c|c|c|c|c|}
\hline $\begin{array}{l}\text { Cuidados paliativos AND } \\
\text { espiritualidade AND Brasil }\end{array}$ & 32 & 11 & 5 & - & 20 & 68 \\
\hline Cuidados paliativos AND relig* & 102 & 24 & 6 & - & - & 132 \\
\hline $\begin{array}{c}\text { Cuidado espiritual AND cuidados } \\
\text { paliativos AND Brasil } \\
\end{array}$ & 52 & 19 & 2 & - & - & 73 \\
\hline Palliative care AND relig* AND Brazil & - & 20 & 5 & 15 & - & 40 \\
\hline $\begin{array}{c}\text { Palliative care AND } \\
\text { spirituality AND Brazil }\end{array}$ & - & 15 & 4 & 12 & - & 31 \\
\hline $\begin{array}{c}\text { Spiritual care AND palliative } \\
\text { care AND Brazil }\end{array}$ & - & 28 & 6 & 21 & - & 55 \\
\hline $\begin{array}{c}\text { Capelão AND Cuidados } \\
\text { Paliativos AND Brasil }\end{array}$ & 5 & 1 & 0 & - & - & 6 \\
\hline $\begin{array}{c}\text { Capelania AND Cuidados } \\
\text { Paliativos AND Brasil } \\
\end{array}$ & 0 & 0 & 0 & - & - & 0 \\
\hline $\begin{array}{c}\text { Chaplaincy and Palliative } \\
\text { Care AND Brazil }\end{array}$ & 31 & - & - & - & - & 31 \\
\hline $\begin{array}{c}\text { Chaplains and Palliative } \\
\text { Care AND Brazil }\end{array}$ & 56 & - & - & - & - & 56 \\
\hline $\begin{array}{c}\text { Cuidados Paliativos AND } \\
\text { Espiritualidade AND } \\
\text { Psicologia AND Brasil } \\
\end{array}$ & - & 64 & 34 & - & - & 98 \\
\hline $\begin{array}{l}\text { Spirituality AND Palliative care } \\
\text { AND Psychology AND Brazil }\end{array}$ & - & - & - & 47 & - & 47 \\
\hline TOTAL & 278 & 182 & 62 & 95 & 20 & 637 \\
\hline
\end{tabular}

Fonte: Elaboração dos autores (2019).

Em congruência com o objetivo proposto, a seleção dos estudos para análise levou em conta os seguintes critérios de inclusão: 1) ter sido desenvolvido no Brasil; 2) ter sido publicado em português e/ou em inglês; 3 ) abordar a E/R no contexto específico dos CP. Ou seja, trabalhos com abordagem sobre espiritualidade junto a pacientes em tratamento oncológico sem referência explícita ao contexto de Cuidados Paliativos não foram incluídos; 4) apresentar resultados sobre $\mathrm{E} / \mathrm{R}$ nos $\mathrm{CP}$, mesmo que a $\mathrm{E} / \mathrm{R}$ não tenha sido o foco central da investigaçáo; 5) ter acesso ao texto completo. Foram excluídos: 1) ensaios teóricos; 2) estudos no contexto de doenças crônicas ou potencialmente de CP, como câncer, doença cardíaca e renal, porém sem referência direta a CP; 3) estudos duplicados, com versão em português e em inglês (foi selecionada apenas uma das versóes), ou uma outra versão de um mesmo estudo, com base na mesma amostra.

\section{Resultados}

A busca nas bases de dados resultou na captura de 637 estudos, incluindo artigos, teses e dissertaçôes. A Tabela 1 apresenta os termos de busca utilizados e o número de estudos encontrados em cada uma dessas bases. Após a primeira avaliação dos estudos 
Figura 1 - Fluxograma do processo de pesquisa e seleçáo dos estudos.

Estudas (artigos, beses e dissertaçōes) idenlíficados nas bases de dadas: Periódicos CAPES; BVS: SCIELO; PUBMED; BDTD. $n=637$

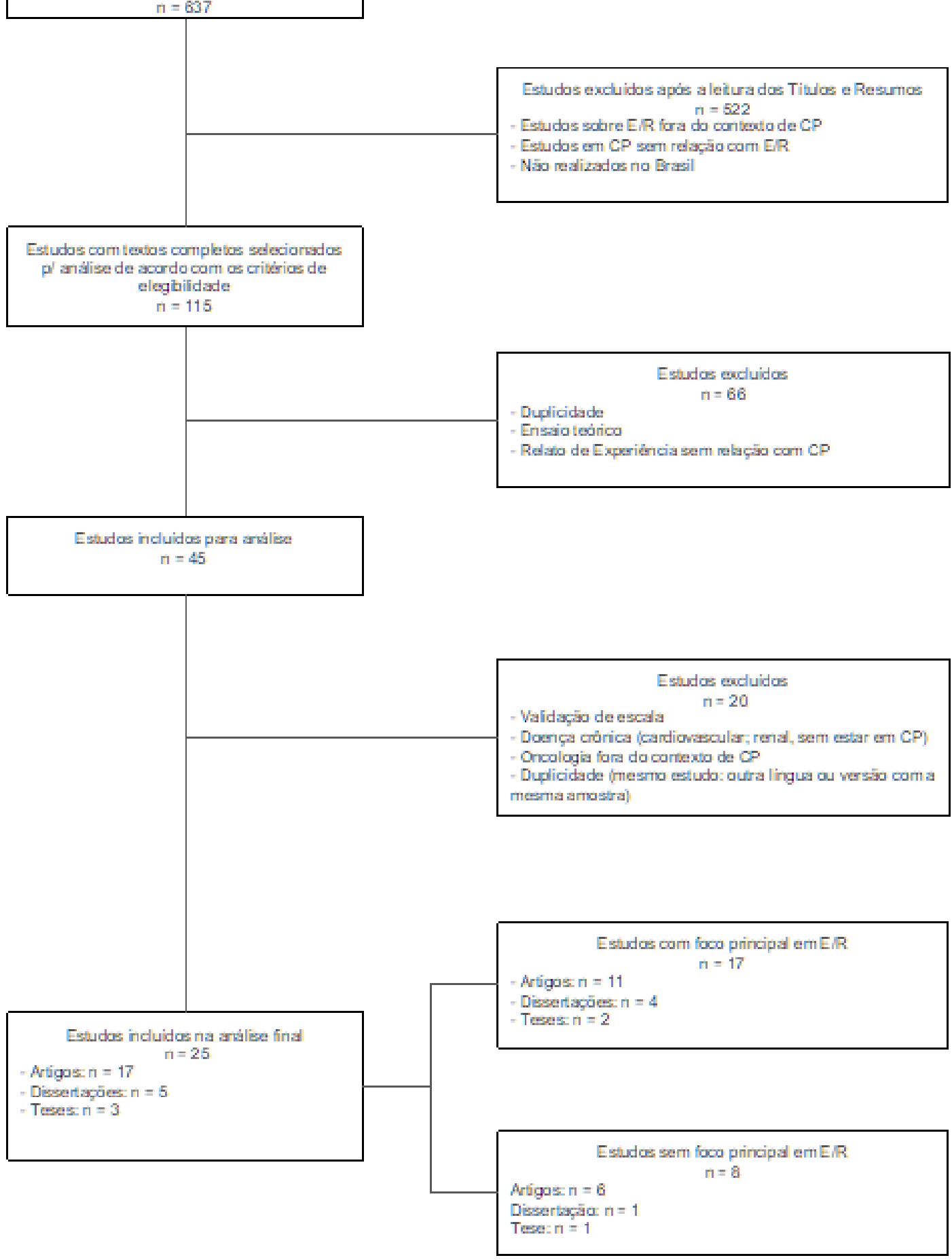

Fonte: Elaboração dos autores (2019). 
capturados, aplicados os critérios de inclusão, 115 foram selecionados para análise. Uma segunda avaliação foi realizada com a participação de um outro revisor. A aplicação dos critérios na seleçáo para análise resultou na inclusão final de 25 estudos, sendo 17 deles com foco principal sobre E/R nos CP (Tabela 2 e 3).

Areas de conhecimento e local geográfico onde os estudos foram desenvolvidos, lingua utilizada nas publicaçôes e abordagem dos estudos

A Enfermagem é a área que apresenta maior número de estudos, sendo dez no total: seis artigos, uma tese e três dissertaçóes, sendo que oito deles tiveram a $\mathrm{E} / \mathrm{R}$ como foco central na investigação. A Medicina vem em seguida e é a que mais tem artigos publicados, sendo cinco deles em inglês. A Psicologia ocupa a terceira posição com cinco estudos (quatro publicados).

Quanto à proveniência geográfica, os estudos sobre $\mathrm{E} / \mathrm{R}$ em $\mathrm{CP}$ têm se restringido a sete Unidades da Federação (Tabela 2 e 3). Sáo Paulo é o Estado com maior número de estudos (sete) com foco central em E/R.

Em relaçáo à língua utilizada nas publicaçóes, oito foram publicados somente em português, cinco em inglês e quatro tiveram publicação bilingue, em português e inglês. Ou seja, 52,9\% do total dos artigos publicados podem ser lidos em língua inglesa. A abordagem qualitativa foi predominante, tendo sido utilizada em $76 \%$ dos estudos (n $=19)$. Cinco estudos $(20 \%)$ privilegiaram uma metodologia quantitativa e apenas um usou o método misto de abordagem.

Tabela 2 - Estudos sobre Espiritualidade/Religiosidade em Cuidados Paliativos.

\begin{tabular}{|c|c|c|c|c|c|}
\hline Área & Artigos & Dissertaçáo & Tese & Local dos estudos & Total \\
\hline Enfermagem & 6 & 3 & 1 & $\begin{array}{l}\text { RS: } 2 \\
\text { SP: } 1 \\
\text { RJ: } 3 \\
\text { PB: } 2 \\
\text { CE: } 1 \\
\text { MG: } 1 \\
\end{array}$ & 10 \\
\hline Medicina & 7 & - & - & $\begin{array}{l}\text { SP: } 5 \\
\text { PE: } 1 \\
\text { RS: } 1 \\
\end{array}$ & 7 \\
\hline Psicologia & 4 & 1 & - & $\begin{array}{l}\text { SP: } 2 \\
\text { PE: } 1 \\
\text { RJ: } 2\end{array}$ & 5 \\
\hline Educação em Saúde & - & - & 1 & RS: 1 & 1 \\
\hline Saúde Coletiva & - & - & 1 & RJ: 1 & 1 \\
\hline Saúde Pública & - & 1 & & RJ: 1 & 1 \\
\hline Total & 17 & 5 & 3 & $\begin{array}{c}\text { 25 } \\
\text { SP: } 8 \\
\text { RJ: } 7 \\
\text { RS: } 4 \\
\text { PB: } 2 \\
\text { PE: } 2 \\
\text { CE: } 1 \\
\text { MG: } 1\end{array}$ & 25 \\
\hline
\end{tabular}

Fonte: Elaboração dos autores (2019). 
Tabela 3 - Estudos sobre Espiritualidade/Religiosidade em Cuidados Paliativos com Foco Central em E/R.

\begin{tabular}{|c|c|c|c|c|c|}
\hline Área & Artigos & Dissertação & Tese & Local dos estudos & Total \\
\hline Enfermagem & 4 & 3 & 1 & $\begin{array}{l}\text { RS: } 1 \\
\text { SP: } 1 \\
\text { RJ: } 2 \\
\text { PB: } 2 \\
\text { CE: } 1 \\
\text { MG: } 1\end{array}$ & 8 \\
\hline Medicina & 5 & - & - & $\begin{array}{l}\text { SP: } 4 \\
\text { PE: } 1\end{array}$ & 5 \\
\hline Psicologia & 2 & 1 & - & $\begin{array}{l}\text { SP: } 2 \\
\text { PE: } 1\end{array}$ & 3 \\
\hline Educação em Saúde & - & - & 1 & RS: 1 & 1 \\
\hline Saúde Coletiva & - & - & - & - & - \\
\hline Saúde Pública & - & - & - & - & - \\
\hline Total & 11 & 4 & 2 & $\begin{array}{c}17 \\
\text { SP: } 7 \\
\text { RJ: } 2 \\
\text { RS: } 2 \\
\text { PB: } 2 \\
\text { PE: } 2 \\
\text { CE: } 1 \\
\text { MG: } 1\end{array}$ & 17 \\
\hline
\end{tabular}

Fonte: Elaboração dos autores (2019).

\section{Evoluçáo da produçáo científica e a noçáo de $E / R$}

Sem estabelecimento pré-determinado de tempo, a busca capturou duas publicaçóes anteriores a 2010. A primeira, em 2003 (Elias, 2003), a segunda, em 2006 (Elias et al., 2007). Do total dos 25 trabalhos analisados, 17 deles (13 artigos, três dissertaçóes, uma tese), 68\%, foram produzidos entre 2014 e 2018. Dentre os 17 artigos científicos, 11 deles tiveram a E/R como foco central (72,7\%). Oito foram publicados a partir de 2015 (Figura 2).

\section{Figura 2 - Evolução da publicação de artigos.}

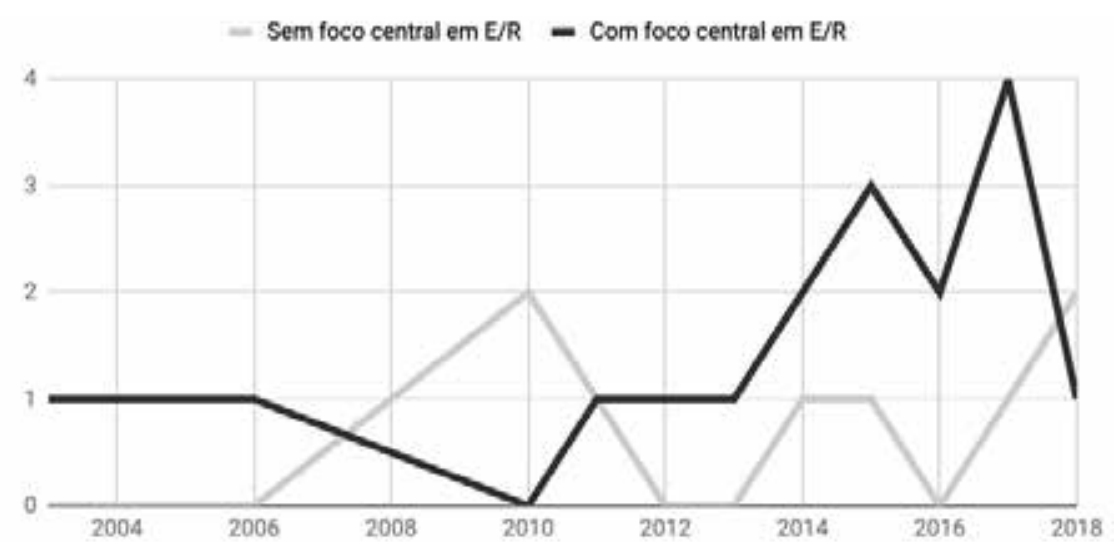

Fonte: Elaboração dos autores (2019). 


\section{Tópicos abordados}

Os 25 estudos selecionados podem ser classificados em três grandes categorias: 1) Papel da E/R nos CP; 2) Modos de integração da E/R nos CP pelos profissionais da saúde; 3) E/R como um "achado" na investigação de outros temas sobre CP.

\section{Papel da E/R no contexto dos CP: fonte de sentido e recurso de coping espiritual/religioso para pacientes e familiares}

Oito estudos investigaram o papel da $\mathrm{E} / \mathrm{R}$ para pacientes e familiares no contexto dos CP. Para pacientes, a $\mathrm{E} / \mathrm{R}$ provê sentido para a experiência de sofrimento, contribui para melhor enfrentamento da morte, provê esperança, apoio (Benites; Neme; Santos, 2017), conforto e fortalecimento (Medeiros, 2012) e melhor qualidade de vida (Camargos et al., 2015; Matos et al., 2017). Esta última foi atrelada a significados de saúde, bem-estar, felicidade e espiritualidade, e permitiu aos autores afirmarem que estratégias de coping espiritual/religioso deveriam ser estimuladas nos pacientes em CP (Matos et al., 2017).

Pesquisadores também encontraram que $90 \%$ de pacientes com câncer avançado reportam conflitos espirituais e buscam na espiritualidade modos de lidar com tais sentimentos (Camargos et al., 2015). Entretanto, "estas necessidades espirituais não são reconhecidas ou são minimamente reconhecidas pelos sistema médico, influenciando potencialmente a qualidade de vida espiritual de modo negativo." (Camargos et al., 2015, p. 13). Os autores observaram ainda que os pacientes apresentaram escores mais elevados de qualidade de vida na dimensão da espiritual, comparados aos escores dos profissionais de saúde (Camargos et al., 2015).

A espiritualidade foi estudada como recurso espiritual e estratégia de coping também no que diz respeito ao uso da oração (Paiva et al., 2014). Pesquisadores investigaram 221 pacientes ambulatoriais com câncer avançado em CP e observaram que escores mais elevados de religiáo estavam associados a níveis mais baixos de inflamação nos pacientes com câncer avançado, em tratamento antineoplásico. Além disso, a "atividade de oração individual foi um fator independente de prognóstico bom em pacientes antineoplásicos ativos" (Paiva et al., 2014, p. 1). Os pesquisadores destacam que a "atividade de oraçâo individual e os escores de religiáo global foram associados à qualidade de vida, sintomas, marcadores infamatórios e sobrevivência" (Paiva et al., 2014, p. 1).

Também para familiares de pacientes em $\mathrm{CP}$, a E/R é fonte para construçáo de sentido e recurso de coping (Paiva et al., 2015; Miqueletto et al., 2017; Rocha, 2017). $\mathrm{A}$ E/R mostrou-se não apenas como recurso de coping no enfrentamento da doença do familiar, mas também como meio para desenvolvimento espiritual, ressignificação da vida e fortalecimento e suporte (Paiva et al., 2015). Entretanto, a despeito de a E/R contribuir na construção de sentido às experiências da vida, as famílias não encontram espaço para a expressão dessas questôes com os profissionais da saúde (Miqueletto et al., 2017). Os autores salientam que essas famílias "reconhecem a E/R como elemento fortalecedor diante das dificuldades enfrentadas pelo adoecimento, mas dependem fortemente da disponibilidade da equipe para incrementar sua experiência" (Miqueletto 
et al., 2017, p. 1616). A espiritualidade beneficia a saúde integral do familiar diante da adversidade própria desse contexto e, neste sentido, não apenas a enfermagem deveria tomar como hábito a provisão de cuidado espiritual aos familiares, como também deveria receber formação acadêmica adequada para tal (Rocha, 2017).

\section{Modos de integração da E/R nos CP pelos profissionais da Saúde}

Nove estudos apresentam resultados sobre modos de integração da E/R nos CP a partir de distintas abordagens e perspectivas: da equipe multiprofissional (Arrieira et al., 2018; Ferreira et al., 2015); de enfermeiros (Evangelista et al., 2016) e residentes da área da saúde (Zaccara, 2014). Arrieira e outros (2018) apontam que os profissionais da equipe de CP reportaram que o cuidado espiritual foi realizado através de açóes tais como oraçáo, oferta de conforto, ajuda no processo de busca de sentido da experiência tanto para os pacientes quanto para a própria equipe multiprofissional.

Estudo realizado com enfermeiros (Evangelista et al., 2016) e residentes da área da saúde (Zaccara, 2014) destacam a falta de formação para a oferta do cuidado espiritual e a dificuldade para identificar as demandas espirituais do paciente, além da insegurança sobre como lidar com essas questóes quando elas emergem. Resende (2014) defende a aplicação da acupuntura para minimizar a "dor espiritual" (ou "dor total"), entendendo o uso dessa técnica como um modo de prover cuidado espiritual.

Três propostas distintas para a prática do cuidado espiritual foram desenvolvidas por Elias (2003), Araújo (2011) e Dezorzi (2016). O estudo pioneiro nesse contexto foi o da psicóloga Ana Catarina Elias (2003), que, juntamente com outros colaboradores (2006; 2007) desenvolveu uma técnica de intervenção denominada RIME: Relaxamento, Imagens Mentais e Espiritualidade, com a finalidade de ressignificar a dor espiritual de pacientes (crianças, adolescentes e adultos) e familiares em CP.

Por meio da pesquisa intervenção, Araújo (2011) desenvolveu, em seu estudo de doutorado, um modelo de cuidado com base em sua própria prática como enfermeiro. O cuidado espiritual foi dirigido a pacientes adultos e fundamentou-se na relação de ajuda (enfermeiro-paciente) e na construção de sentido no sofrimento.

Quanto à proposta de formaçáo de profissionais para a prática do cuidado espiritual em CP, esta foi aplicada pela enfermeira Luciana Dezorzi (2016) em seu estudo de doutorado. O Módulo de Educação sobre espiritualidade foi desenvolvido para profissionais da saúde envolvidos em CP (mas não restrito a este grupo) e publicado como uma cartilha em formato de e-book (Dezorzi; Raymundo; Goldim, 2016). A autora afirma que houve resultados positivos da intervenção educativa, e que "o uso de um módulo de educação pode colaborar para minimizar as lacunas deixadas ao longo do processo formação" (Dezorzi, 2016, p. 65).

\section{E/R como um "achado" na investigação de outros temas sobre CP}

Embora a investigação da espiritualidade não tenha sido o foco principal de oito estudos, esta surgiu como um "achado". Em tais estudo, os pesquisadores estavam interessados em demonstrar: 1) a atuação dos enfermeiros junto à criança em CP (Monteiro et al., 2014); 
2) o processo de morte e morrer da pessoa com câncer em final de vida (Tomaszewski et al., 2017); 3) a experiência de cuidadores de familiares em CP (Lima; Machado, 2018; Rezende et al., 2010); 4) a comunicação (Geovanini, 2011) e a percepção de vários elementos implicados na experiência de final de vida de paciente em CP (Carvalho et al., 2019; Castro; Barreto, 2015; Marinho, 2010). Os resultados apontaram que enfermeiros preocupam-se em oferecer apoio espiritual, emocional e religioso à criança em CP (Monteiro et al., 2014); que a espiritualidade é fonte de produçáo de sentido (Tomaszewski et al., 2017), recurso de enfrentamento (Lima; Machado, 2018) e fonte de produção de maior bem-estar (Rezende et al., 2010) para pacientes e familiares. Os estudos mostraram ainda que a espiritualidade do próprio médico tem influência na forma como a comunicaçáo é realizada com o paciente (Geovanini, 2011), bem como na percepção do sofrimento espiritual (Castro; Barreto, 2015). Os profissionais têm dificuldade de reconhecer e, consequentemente, de atender as necessidades espirituais do paciente (Marinho, 2010). Carvalho e outros (2019, p. 1) mostraram que "menos de $15 \%$ dos pacientes tiveram suas necessidades espirituais atendidas e/ou receberam apoio psicológico" e, segundo Marinho (2010, p. 129) não há avaliação do sofrimento spiritual por parte da equipe, sendo a dimensão da espiritualidade confundida com religiosidade e, portanto, "de caráter privado" (Marinho, 2010, p. 131).

\section{Discussáo}

Há consenso, nas pesquisas brasileiras, sobre o uso e significado do termo espiritualidade?

Dentre os 17 estudos cujo foco central foi a investigaçáo da E/R nos CP, 14 (82,3\%) apresentaram uma definição de espiritualidade. Os pesquisadores brasileiros parecem seguir a tendência internacional na assunção de um conceito de espiritualidade que enfatiza a dimensão do sentido e propósito da vida, ancorando tal definição (com citação direta, indireta ou fonte secundária) em reconhecidos pesquisadores norte-americanos que trabalham no campo da Espiritualidade e Saúde, tais como o psiquiatra Harold Koenig; King; Carson (2012); Crystal Park (2013), psicóloga da religiáo e a médica de medicina interna Christina Puchalski e outros (2014).

$\mathrm{Na}$ definição de espiritualidade, a maioria dos/as pesquisadores/as brasileiros/as faz a diferenciação entre espiritualidade e religiosidade, como fica evidenciado em 70,5\% $(\mathrm{n}=12)$ dos estudos. Essa diferenciação começa a aparecer de modo explícito a partir de 2010. Os dois estudos anteriores a 2010 apresentam uma definição de espiritualidade com maior ênfase à conexáo intrapessoal e transcendental.

Há consenso sobre o significado de cuidado espiritual, bem como um protocolo ou recomendaçóes sobre como este pode ser integrado pelas equipes multidisciplinares de CP?

Apesar de o termo "cuidado espiritual" ter sido mencionado em $64,7 \%(\mathrm{n}=11)$ dos 17 estudos que tiveram a E/R como foco central nos CP, o mesmo foi definido em 
apenas três trabalhos: na dissertação de Resende (2014) e nas teses de Araújo (2011) e Dezorzi (2016). O termo aparece como "sinônimo de cuidado transpessoal" (Resende, 2014, p. 11 - nota de rodapé), como "essencialmente relacional” (Araújo, 2011, p. 128), mas "tem como foco central a busca e a descoberta do sentido da vida, para uma realização plena e para o fim do desespero"(Araújo, 2011, p. 128), e como o cuidado "que reconhece e responde às necessidades do espírito humano, especialmente quando confrontado com traumas, problemas de saúde ou tristeza” (Dezorzi, 2016, p. 31). Dezorzi observa que o cuidado espiritual "começa com o contato humano encorajador e com um relacionamento compassivo, capaz de se mover na direçáo que a necessidade requer" (2016, pp. 31-32).

Em artigos com foco central em CP publicados em periódicos nacionalmente reconhecidos, o aparecimento inicial do termo surgiu no estudo publicado em 2015 (Ferreira et al., 2015). À parte do contexto dos CP, o termo aparece pela primeira vez em periódico nacionalmente reconhecido em 2009, conforme mostra o estudo sobre cuidado espiritual interdisciplinar, de Hefti e Esperandio (2016). Constata-se, portanto, que no cenário dos $\mathrm{CP}$ no Brasil, a noção de cuidado espiritual não apenas é bastante recente, como também sua reflexão teórica é ainda incipiente. Essa realidade evidencia a ênfase biomédica da formação profissional (Marinho, 2010), produzindo, consequentemente, uma compreensão reducionista de espiritualidade, por vezes tomada unicamente em sua forma de expressão religiosa. Essa postura traz dificuldades para identificar, compreender e lidar com o forte papel das crenças espirituais/religiosas nos CP. Contudo, nesses casos, os profissionais argumentam que a religiosidade é uma questão de foro íntimo, portanto, não é direito do profissional da saúde abordar tais questóes (Esperandio; Machado, 2018; Marinho, 2010).

Nenhum dos estudos chegou a discutir a questão de protocolo sobre cuidado espiritual ou fazer recomendaçóes práticas para além de enfatizar a necessidade de formação para uma prática que também contemple as necessidades espirituais de pacientes e familiares em CP.

\section{A quem cabe o cuidado espiritual nos Cuidados Paliativos?}

Essa questão não foi discutida em qualquer dos trabalhos. Também não é mencionada a figura do capeláo, ou da pessoa especialista em cuidado espiritual (conhecida como spiritual caregiver, na literatura internacional) ou outro termo que se refira à pessoa que exerça a função de especialista em assistência espiritual. Também chama a atenção o fato de não ter sido encontrado nenhum estudo no Brasil sobre capelania no contexto dos CP. Algumas buscas não resultaram em captura de trabalhos sobre "capelania e cuidados paliativos", ou "cuidado espiritual e cuidados paliativos". Os estudos publicados sobre capelania hospitalar não foram desenvolvidos no cenário dos CP.

Embora vários estudos mencionem o termo "cuidado espiritual", percebe-se não haver suficiente clareza sobre seu sentido e aplicação. Talvez esta seja uma das razóes que expliquem o fato de os estudos não abordarem se o cuidado espiritual é algo a ser praticado por toda a equipe de CP; de que modo este deve ser realizado; ou se seria prerrogativa de alguma especialidade. 
É consenso na literatura internacional (Puchalski et al. 2014; Leget, 2018) que o cuidado espiritual, sendo expressão do atendimento das necessidades espirituais de pacientes e familiares, é essencialmente interdisciplinar. Neste sentido, uma triagem de tais necessidades pode ser realizada por qualquer um dos profissionais da equipe (Puchalski et al., 2014; Hefti; Esperandio, 2016). Entretanto, é responsabilidade do/a cuidador/a espiritual avaliar melhor as necessidades espirituais (seja dos pacientes ou de sua família) e atender aos casos que exijam sua especialidade como tal. $\mathrm{O}$ trabalho competente deste/a profissional para compor a equipe de CP é de grande contribuição. Por exemplo, em situaçôes de tomada de decisão onde as crenças espirituais/religiosas têm predominância, pacientes demonstram confiança e abertura para discussão das opçóes de tratamento com o/a assistente espiritual especializado/a. Profissionais da saúde constatam a força das crenças religiosas em tomadas de decisão em saúde, por exemplo, na sedação paliativa (Eich et al., 2018), e mesmo em tratamentos invasivos (Elliott et al., 2012; Maessen et al., 2009; Johnson et al., 2016).

No Brasil, apesar da religião ter um papel central na vida da maioria dos brasileiros, como mostram os estudos de Huber; Huber (2012) e de Esperandio e outros (2019), em geral, os hospitais não têm a tradição de ter em seus quadros de funcionários, a vaga para o trabalho do/a capelão/ã, senáo com raras exceçóes. A capelania no Brasil funciona de modo voluntário, fragmentado, com pouco diálogo inter-religioso no contexto hospitalar (Gentil; Guia; Sanna, 2011) e com insuficiente preparo de capeláes/âs para atuação na assistência espiritual em CP. Com esse cenário, a participação desse profissional em uma equipe multidisciplinar torna-se rara. Em situações ocasionais e específicas, o líder espiritual do paciente e da família é contactado por alguém da equipe multidisciplinar.

\section{As pesquisas empiricas sobre E/R em CP estão teoricamente ancoradas?}

A implementaçáo de serviços em CP no Brasil, bem como os estudos sobre E/R nesse contexto estáo apenas em seu início, mas em franco crescimento. $\mathrm{O}$ aumento no volume de pesquisas sobre o tema e a implementação de CP no Brasil parece estar caminhando em paralelo pois ambos começam a ganhar mais visibilidade nos últimos 4 anos, entre 2015 e 2018.

Se por um lado esse aumento ainda náo demonstra significativo aprofundamento ou avanço no conhecimento teórico-prático sobre o tema, por outro lado, evidencia o interesse e necessidade de pesquisas. Parece haver em vários estudos, preocupação em comprovar a relação (geralmente positiva) entre espiritualidade e resultados em saúde, destacando consequentemente, a relevância dessa integração. Mas há carência de proposiçãa, aplicação e avaliaçáo de modelos de cuidado espiritual. Debates de consenso nacional, a exemplo do que ocorreu nos Estados Unidos e Europa sobre o significado de espiritualidade e da importância da prática de cuidado espiritual na saúde, fazem-se necessários no Brasil.

Poucos estudos empíricos apresentam um quadro de referência teórico para apoiar o levantamento e análise dos dados. As questóes teóricas são, portanto, uma lacuna a ser preenchida em futuros estudos, sobretudo, considerando a rápida expansão na implementação de serviços de $\mathrm{CP}$ e a necessidade de que tais serviços estejam teoricamente 
bem fundamentados, incluindo o cuidado espiritual como um de seus componentes essenciais (Puchalski et al., 2014; Nolan; Saltmarsh; Leget, 2011).

\section{Conclusão}

Em síntese, os estudos mostram que a investigaçáo sobre $\mathrm{E} / \mathrm{R}$ no contexto dos $\mathrm{CP}$ é bastante recente, em crescimento a partir de 2015, e vem ocorrendo em paralelo à progressão na oferta de serviços de CP. Isto parece indicar que o Brasil tem um grande potencial para o desenvolvimento de estudos sobre cuidado espiritual nos CP. Há consenso entre os pesquisadores sobre a noção de espiritualidade como sendo mais ampla e distinta de religiosidade e referida às questóes de sentido e propósito. Entretanto, um consenso nacional quanto a definição de espiritualidade e cuidado espiritual ainda se faz necessário. Tal consenso poderá contribuir, tanto para o avanço do conhecimento teórico-prático no campo do Cuidado Ético, quanto para nortear a organização (e implementaçáo) do cuidado espiritual como serviço especializado e integrado às equipes multidisciplinares de Cuidados Paliativos.

Em relação ao cuidado espiritual propriamente dito, este tem sido muito pouco investigado, sua noção não é clara, os raros modelos disponíveis ainda não têm sido suficientemente testados, não há protocolo nem treinamento disponível e específico para a prática nos CP, e é dependente da boa vontade, intuição e da própria espiritualidade do profissional da saúde. Todavia, muitos profissionais da saúde, de forma mais frequente na área da enfermagem, valorizam o cuidado espiritual e procuram integrá-lo na prática de cuidado, ainda que intuitivamente e sem nenhuma formação.

Conclui-se que embora a espiritualidade seja uma dimensão fundante na constituição da subjetividade humana (e quando esta se expressa, predominantemente, em sua expressão religiosa ocupe posição central na vida do indivíduo), esta não tem sido plena e devidamente integrada nos serviços de CP no Brasil. A manifestação de seus aspectos, quer seja na forma de recurso positivo ou de necessidade a ser atendida, é muitas vezes ignorada, minimizada, desvalidada e/ou náo identificada pelos profissionais do cuidado em saúde. A equipe multidisciplinar tem, em geral, dificuldades variadas e em diferentes níveis, para lidar com conteúdos próprios dessa dimensão.

Há, portanto, carência na formação profissional; na proposição de protocolos e de possíveis modelos de cuidado espiritual; e ainda, de estudos empíricos teoricamente embasados que aprofundem as questóes próprias da E/R no contexto dos CP. Também há carência de estudos tanto no que diz respeito à assistência espiritual de pacientes, familiares e equipe multidisciplinar, quanto em relação ao modo como a E/R impacta nas tomadas de decisáo em saúde e no processo de enfrentamento de uma doença grave e ameaçadora da continuidade da existência. Diante disso, pode-se afirmar que essa área de conhecimento tem um grande potencial a ser desenvolvido e é significativo o número de pesquisadores/as interessados/as em fomentar esse campo.

Por fim, em razão das bases de dados utilizadas e dos critérios de inclusão/exclusão estabelecidos, alguns trabalhos podem não ter sido capturados nesse levantamento, sendo, portanto, uma limitação possível do presente estudo. 


\section{Referências}

ACADEMIA NACIONAL DE CUIDADOS PALIATIVOS. Onde existem. Disponível em: <https://paliativo.org.br/ancp/onde-existem/>. Acesso em: 23 nov. 2018.

ARAUJO, Michell Ângelo Marques. O cuidado espiritual: um modelo à luz da análise existencial e da relação de ajuda. Tese (Doutorado em Enfermagem) Fortaleza (CE): Universidade Federal do Ceara, Faculdade, 2011.

ARRIEIRA, Isabel Cristina De Oliveira et al. Spirituality in palliative care: experiences of an interdisciplinary team. Revista da Escola de Enfermagem da USP, v. 52, p. $1-8,2018$.

BENITES, Andréa Carolina; NEME, Carmen Maria Bueno; SANTOS, Manoel Antonio dos. Significados da espiritualidade para pacientes com câncer em cuidados paliativos. Estudos de Psicologia (Campinas), v. 34, n. 2, p. 269-279, jun. 2017.

BRASIL. Resolução No 41, de 31 de outubro de 2018. Dispóe sobre as diretrizes para a organizaçáo dos cuidados paliativos, à luz dos cuidados continuados integrados, no âmbito Sistema Único de Saúde (SUS). Diário Oficial da União: seção 1, Brasília, DF, No 225, p. 275, 23 nov. 2018.

CAMARGOS, Mayara Goulart et al. Understanding the Differences Between Oncology Patients and Oncology Health Professionals Concerning Spirituality/ Religiosity: A Cross-Sectional Study. Medicine, v. 94, n. 47, p. 1-15, 1 nov. 2015.

CARVALHO, Guilherme D. et al. The Quality of End-of-Life Care after Limitations of Medical Treatment as Defined by a Rapid Response Team: A Retrospective Cohort Study. Journal of Palliative Medicine, v. 22, n. 1, p. 71-74, jan. 2019.

CASTRO, Elisa Kern de; BARRETO, Silvia Menna. Critérios de Médicos Oncologistas para Encaminhamento Psicológico em Cuidados Paliativos. Psicologia: Ciência e Profissão, v. 35, n. 1, p. 69-82, mar. 2015.

DEZORZI, Luciana Winterkorn. Espiritualidade na atenção a pacientes em cuidados paliativos e os processos de educação dos profissionais de saúde. Tese (Doutorado em Enfermagem). Porto Alegre - RS: Universidade Federal do Rio Grande do Sul, Programa de Pós-Graduação em Medicina: Ciências Médicas, Faculdade de Medicina, 2016.

DEZORZI, Luciana Winterkorn; RAYMUNDO, Marcia Mocellin; GOLDIM, José Roberto. Espiritualidade na atenção a pacientes/famílias em cuidados paliativos. Disponível em: <https://issuu.com/nucleointerdisciplinardebioetica/docs/ espiritualidade_na_aten___o_a_paci>. Acesso em: 2 jun. 2019.

EICH, Melisse et al. Princípios e valores implicados na prática da sedação paliativa e a eutanásia. Interface - Comunicação, Saúde, Educaçãoo, v. 22, n. 66, p. 733-744, set. 2018. 
ELIAS, Ana Catarina de Araújo et al. Training program about the therapeutical intervention "relaxation, mental images and spirituality" (RIME) to resignify the spiritual pain of terminal patients. Archives of Clinical Psychiatry (São Paulo), v. 34, p. 60-72, 2007.

ELIAS, Ana Catarina de Araújo. Re-significação da dor simbólica da morte: relaxamento mental, imagens mentais e espiritualidade. Psicologia: Ciência e Profissão, v. 23, n. 1, p. 92-97, mar. 2003.

ELIAS, Ana Catarina de Araújo et al. Therapeutical intervention, relaxation, mental images, and spirituality (RIME) for spiritual pain in terminal patients. A training program. The Scientific World Journal, v. 6, p. 2158-2169, 27 jun. 2006.

ELLIOTT, Barbara A. et al. Religious Beliefs and Practices in End-Stage Renal Disease: Implications for Clinicians. Journal of Pain and Symptom Management, v. 44, n. 3, p. 400-409, set. 2012.

ESPERANDIO, Mary Rute Gomes et al. Brazilian Validation of Centrality of Religiosity Scale (CRS-10BR and CRS-5BR). Religions, v. 10, n. 9, p. 508, 30 ago. 2019 .

ESPERANDIO, Mary Rute Gomes; MACHADO, Geilson Antonio Silva. Brazilian Physicians' Beliefs and Attitudes Toward Patients' Spirituality: Implications for Clinical Practice. Journal of Religion and Health, p. 1-16, 29 set. 2018.

EVANGELISTA, Carla Braz et al. Espiritualidade no cuidar de pacientes em cuidados paliativos: Um estudo com enfermeiros. Escola Anna Nery, v. 20, n. 1, p. 176-182, mar. 2016.

FERREIRA, Alberto Gorayeb de Carvalho et al. Concepções de Espiritualidade e Religiosidade e a Prática Multiprofissional em Cuidados Paliativos. Revista Kairós Gerontologia, v. 18, n. 3, p. 227-244, jul. 2015.

GENTIL, Rosana Chami; GUIA, Beatriz Pinheiro da; SANNA, Maria Cristina Organização de serviços de capelania hospitalar: um estudo bibliométrico. Escola Anna Nery, v. 15, n. 1, p. 162-170, mar. 2011.

GEOVANINI, Fátima. Notícias Que (Des) Enganam: O Impacto da Revelação do Diagnóstico e as Implicaçóes Éticas na Comunicação de Más Notícias para Pacientes Oncológicos. Dissertação (Mestrado em Saúde Pública). Rio de Janeiro: Fundação Osvaldo Cruz. Escola Nacional de Saúde Pública., 2011.

HEFTI, René; ESPERANDIO, Mary Rute Gomes. The Interdisciplinary Spiritual Care Model - A holistic Approach to Patient Care. HORIZONTE, v. 14, n. 41, p. 13, 31 mar. 2016.

HUBER, Stefan; HUBER, Odilo W. The Centrality of Religiosity Scale (CRS). Religions, v. 3, n. 3, p. 710-724, 20 ago. 2012.

INSTITUTO BRASILEIRO DE GEOGRAFIA E ESTATÍSTICA - IBGE. Número de idosos cresce 18\% em 5 anos e ultrapassa 30 milhóes em 2017. 
Disponível em: <https://agenciadenoticias.ibge.gov.br/agencia-noticias/2012agencia-de-noticias/noticias/20980-numero-de-idosos-cresce-18-em-5-anos-eultrapassa-30-milhoes-em-2017>. Acesso em: 13 jan. 2020.

JOHNSON, Jerry et al. The Impact of Faith Beliefs on Perceptions of End-of-Life Care and Decision Making among African American Church Members. Journal of Palliative Medicine, v. 19, n. 2, p. 143-148, fev. 2016.

KASTNER, Monika et al. What is the most appropriate knowledge synthesis method to conduct a review? Protocol for a scoping review. BMC Medical Research Methodology, v. 12, n. 1, p. 114, dez. 2012.

KOENIG, Harold G.; KING, Danna E.; CARSON, Verna Benner. Handbook of religion and health. 2nd ed ed. Oxford ; New York: Oxford University Press, 2012.

LEGET, Carlo. Spirituality in Palliative Care. In: MACLEOD, R. D.; VAN DEN BLOCK, L. (Eds.). Textbook of Palliative Care. Cham: Springer International Publishing, 2018. p. 1-11.

LIMA, Carolina Peres de; MACHADO, Mariana de Abreu. Cuidadores Principais Ante a Experiência da Morte: Seus Sentidos e Significados. Psicologia: Ciência e Profissão, v. 38, n. 1, p. 88-101, mar. 2018.

MAESSEN, Maud. et al. Trends and determinants of end-of-life practices in ALS in the Netherlands. Neurology, v. 73, n. 12, p. 954-961, 22 set. 2009.

MARINHO, S. OLIVEIRA. Cuidados paliativos e práticas de saúde: um estudo sobre a gestáo da morte na sociedade contemporânea. Tese (Doutorado em Saúde Coletiva) - Rio de Janeiro: Instituto de Medicina Social, Universidade do Estado do Rio de Janeiro, 2010.

MATOS, Ticiane Dionizio de Sousa et al. Qualidade de vida e coping religiosoespiritual em pacientes sob cuidados paliativos oncológicos. Revista LatinoAmericana de Enfermagem, v. 25, p. 1-9, 2017.

MEDEIROS, Waleska de Carvalho Marroquim. A clínica psicológica e a experiência da espiritualidade de pacientes em cuidados paliativos. Dissertação (Mestrado em Psicologia) Recife (PE): Universidade Catolica de Pernambuco. Programa de PósGraduação em Psicologia Clinica, 2012.

MIQUELETTO, Marcelo et al. Espiritualidade de famílias com um ente querido em situação de final de vida. Revista Cuidarte, v. 8, n. 2, p. 1616-27, 1 maio 2017.

MONTEIRO, Ana Claudia Moreira et al. A atuação do enfermeiro junto à criança com câncer: cuidados paliativos. Revista Enfermagem UERJ, v. 22, n. 6, p. $778-$ 783, 23 dez. 2014.

NOLAN, Steve; SALTMARSH, Philip; LEGET, Carlo. Spiritual care in palliative care: Working towards an EAPC task force. European Journal of Palliative Care, $p$. 86-89, 2011. 
Espiritualidade em cuidados paliativos no Brasil...

PAIVA, Bianca Sakamoto Ribeiro et al. "Oh, yeah, I'm getting closer to god": spirituality and religiousness of family caregivers of cancer patients undergoing palliative care. Supportive Care in Cancer, v. 23, n. 8, p. 2383-2389, ago. 2015.

PAIVA, Carlos Eduardo et al. The Impact of Religiosity and Individual Prayer Activities on Advanced Cancer Patients' Health: Is There Any Difference in Function of Whether or Not Receiving Palliative Anti-neoplastic Therapy? Journal of Religion and Health, v. 53, n. 6, p. 1717-1727, dez. 2013.

PARK, Crystal L. Religion and Meaning. In: PALOUTZIAN, Raymond F.; PARK, Crystal L. (Eds.). Handbook of the psychology of religion and spirituality. Second edition ed. New York London: The Guilford Press, 2013. p. 295-314.

PUCHALSKI, Cristina M. et al. Improving the Spiritual Dimension of Whole Person Care: Reaching National and International Consensus. Journal of Palliative Medicine, v. 17, n. 6, p. 642-656, jun. 2014.

RESENDE, Thais Mendonça. Acupuntura para Pacientes Oncológicos Ambulatoriais: Um Cuidado Espiritual de Enfermagem. Dissertação (Mestrado em Enfermagem). Programa de Pós-Graduação em Enfermagem - Juiz de Fora: Faculdade de Enfermagem. Universidade Federal de Juiz de Fora, 2014.

REZENDE, Vera Lucia et al. Avaliação psicológica dos cuidadores de mulheres com câncer pelo General Comfort Questionnaire. Paidéia (Ribeirão Preto), v. 20, n. 46, p. 229-237, ago. 2010.

ROCHA, Renata Carla Nencetti Pereira. Experiências e necessidades espirituais do familiar cuidador de paciente em atenção paliativa oncológica. Dissertação (Mestrado Profissional em Enfermagem Assistencial) - Niterói: Universidade Federal Fluminense - Escola de Enfermagem Aurora de Afonso Costa, 2017.

SOUZA, Marcela Tvares de; SILVA, Michelly Dias da; CARVALHO, Rachel de. Integrative review: what is it? How to do it? Einstein (São Paulo), v. 8, n. 1, p. 102-106, mar. 2010.

TOMASZEWSKI, Adriana Soares et al. Manifestaçóes e necessidades referentes ao processo de morte e morrer: perspectiva da pessoa com câncer Demonstrations and necessities on the death and dying process: perspective of the person with cancer. Revista de Pesquisa: Cuidado é Fundamental Online, v. 9, n. 3, p. 705, 11 jul. 2017.

WORLDWIDE PALLIATIVE CARE ALLIANCE (WPCA); WORLD HEALTH ORGANIZATION (WHO). Global Atlas of Palliative Care at the End of Life. London: WPCA, 2014.

ZACCARA, Ana Aline Lacet. Cuidados Paliativos e Espiritualidade: estudo com residentes da área da Saúde. Dissertação (Mestrado em Enfermagem) - João Pessoa: Universidade Federal da Paraíba, 2014.

Recebido: 20 de janeiro de 2020 .

Aprovado: 13 de maio de 2020. 